\title{
On Noncommutative Corrections of Gravitational Energy in Teleparallel Gravity
}

\author{
S. C. Ulhoa ${ }^{1,2}$ and R. G. G. Amorim ${ }^{1,2}$ \\ ${ }^{1}$ Instituto de Física, Universidade de Brasília, 70910-900 Brasília, DF, Brazil \\ ${ }^{2}$ Faculdade Gama, Universidade de Brasília, Setor Leste (Gama), 72444-240 Brasília, DF, Brazil \\ Correspondence should be addressed to S. C. Ulhoa; sc.ulhoa@gmail.com
}

Received 27 February 2013; Accepted 26 May 2013

Academic Editor: Shinji Tsujikawa

Copyright (c) 2013 S. C. Ulhoa and R. G. G. Amorim. This is an open access article distributed under the Creative Commons Attribution License, which permits unrestricted use, distribution, and reproduction in any medium, provided the original work is properly cited.

\begin{abstract}
We use the theory of teleparallelism equivalent to general relativity based on noncommutative spacetime coordinates. In this context, we write the corrections of the Schwarzschild solution. We propose the existence of a Weitzenböck spacetime that matches the corrected metric tensor. As an important result, we find the corrections of the gravitational energy in the realm of teleparallel gravity due to the noncommutativity of spacetime. Then we interpret such corrections as a manifestation of quantum theory in gravitational field.
\end{abstract}

\section{Introduction}

The notion of noncommutative spatial coordinates arose with Heisenberg, who wrote a letter to Peierls, in 1930, about the existence of an uncertain relation between coordinates in space-time as a possible solution to avoid the singularities in the self-energy terms of pontual particles. Based on such an advice, Peierls applied those ideas on the analysis of the Landau problem which can be described by an electric charge moving into a plane under the influence of a perpendicular magnetic field. Since then, Peierls commented about it with Pauli, who included Oppenheimer in the discussion. Oppenheimer presented the ideas to Hartrand Snyder, his former Ph.D. student [1-3]. Thus Snyder was the first to discuss the idea that spatial coordinates could not commutate to each other at small distances which is a change of perspective of tiny scales $[4,5]$. It is worth recalling that the concept of noncommutativity itself is not new in Physics; in fact in Quantum Mechanics the uncertain principle, which is a noncommutative relation between coordinates and momenta, plays a fundamental role. Therefore at the beginning, with the pioneer works of Snyder, the idea was to use the noncommutativity between spacetime coordinates to control the ultraviolet divergences into the realm of quantum electrodynamic. Such an approach, however, got into oblivion due to the success achieved by the so-called renormalization process. More recently the interest of the physical community resurfaced with the application of noncommutative geometry in nonabelian theories [6], in gravitation [7-9], in standard model [10-12], and in the problem of the quantic Hall effect [13]. Certainly the discover that the dynamics of an open string can be explained by noncommutative gauge theories at specific limits [14] has contributed to this renewed interest of the scientific community in the topic.

From the mathematical point of view, the simplest algebra of the Hermitian operators $\widehat{x}^{\mu}$, whose mean values correspond to observable coordinates, is given by

$$
\left[\widehat{x}^{\mu}, \widehat{x}^{\nu}\right]=i \theta^{\mu \nu}
$$

where $\theta^{\mu \nu}$ is an antisymmetric constant tensor. From the above expression it is possible to obtain the following uncertain relations

$$
\Delta \widehat{x}^{\mu} \Delta \widehat{x}^{v} \geq \frac{1}{2}\left|\theta^{\mu v}\right|
$$

which suggest that effects due to the noncommutativity into spacetime turn out to be relevant at scales of the order 
of $\sqrt{\left|\theta^{\mu \nu}\right|}$. Usually the noncommutativity is introduced by means of useing the Moyal product [15] defined as

$$
\begin{aligned}
f(x) \star g(x) \equiv & \left.\exp \left(\frac{i}{2} \theta^{\mu \nu} \frac{\partial}{\partial x^{\mu}} \frac{\partial}{\partial y^{\nu}}\right) f(x) g(y)\right|_{y \rightarrow x} \\
= & f(x) g(x)+\frac{i}{2} \theta^{\mu \nu} \partial_{\mu} f \partial_{\nu} g \\
& +\frac{1}{2 !}\left(\frac{i}{2}\right)^{2} \theta^{\mu_{1} \nu_{1}} \theta^{\mu_{2} \nu_{2}}\left(\partial_{\mu_{1}} \partial_{\mu_{2}} f\right)\left(\partial_{\nu_{1}} \partial_{\nu_{2}} g\right) \\
& +\cdots
\end{aligned}
$$

with a constant $\theta^{\mu \nu}$. The procedure used for the construction of field theory in noncommutative coordinates is to write the actions replacing the usual product, in the classical lagrangian density, by the Moyal product. In this way, using the properties of noncommutative algebra, we can see that a free field theory has the action of noncommutative field equal to the action of the field in ordinary coordinates. Moreover, in the presence of interactions, effects of noncommutativity are quite significant [14].

An alternative theory of gravitation is the so-called teleparallel gravity which was introduced by Einstein, as an attempt to unify gravity and electromagnetic field [16], and Cartan, who developed the main part of the theory [17]. From the dynamics point of view, teleparallel gravity and general relativity predict the same results. On the other hand, teleparallel gravity allows for the definition of quantities that are physically of interest, such as the gravitational energy momentum and angular momentum tensors which are well behaved [18] when compared to attempts made in the context of general relativity $[19,20]$. In addition, none of such expressions obtained in the realm of general relativity are dependant on the reference frame, which is certainly not a desirable feature for energy, momentum, and angular momentum.

The expressions for the energy momentum and angular momentum of the gravitational field, in the context of the Teleparallelism Equivalent to General Relativity (TEGR), are invariant under transformations of the coordinates of the three-dimensional spacelike surface; they are also dependent on the frame of reference, as is to be expected. They have been applied consistently over the years for many different systems [21-25]. The frame dependence is an expected condition for any expression due to the field since in special relativity the energy of a particle for a stationary observer is $m$ (with $c=1$ ), but it is $\gamma m$ for an observer under a Lorentz transformation boost (here $\gamma$ is the Lorentz factor). There is no reason to abandon this feature once dealing with the gravitational field, and similar behaviour is expected for momentum and angular momentum.

Therefore we have two successful theories described above and a natural forward step is to combine both of them. Then here our aim is to study the Teleparallelism Equivalent to General Relativity in the noncommutative spacetime context. In Section 2 we introduce the concepts of teleparallel gravity, giving the definition of gravitational energy. In this way, in Section 3 we present the corrections of the Schwarzschild solution due to the noncommutativity of tetrad fields and the respective correction in the gravitational energy of the whole spacetime. Finally in last section we address our concluding remarks.

Notation. Spacetime indices $\mu, \nu, \ldots$ and $\mathrm{SO}(3,1)$ indices $a, b, \ldots$ run from 0 to 3 . Time and space indices are indicated according to $\mu=0, i, a=(0),(i)$. The tetrad field is denoted by $e^{a}{ }_{\mu}$ and the determinant of the tetrad field is represented by $e=\operatorname{det}\left(e^{a}{ }_{\mu}\right)$.

\section{Teleparallel Gravity}

The Teleparallelism Equivalent to General Relativity (TEGR) is constructed out of tetrad fields (instead of a metric tensor) in the Weitzenböck (or Cartan) spacetime, in which it is possible to have distant (or absolute) parallelism [16]. The tetrad field and metric tensor are related by

$$
\begin{aligned}
& g^{\mu \nu}=e^{a \mu} e_{a}^{\nu}, \\
& \eta^{a b}=e^{a \mu} e^{b},
\end{aligned}
$$

where $\eta^{a b}=\operatorname{diag}(-+++)$ is the metric tensor of Minkowski spacetime. Then, the Minkowski spacetime metric tensor raises and lowers tetrad indices, similar to the metric tensor in curved spacetime.

Let us start with a manifold endowed with a Cartan connection [17], $\Gamma_{\mu \lambda \nu}=e^{a}{ }_{\mu} \partial_{\lambda} e_{a v}$, which can be written as

$$
\Gamma_{\mu \lambda \nu}={ }^{0} \Gamma_{\mu \lambda \nu}+K_{\mu \lambda \nu}
$$

where ${ }^{0} \Gamma_{\mu \lambda \nu}$ are the Christoffel symbols and $K_{\mu \lambda \nu}$ is given by

$$
K_{\mu \lambda \nu}=\frac{1}{2}\left(T_{\lambda \mu \nu}+T_{\nu \lambda \mu}+T_{\mu \lambda \nu}\right)
$$

$K_{\mu \lambda \nu}$ is the contortion tensor defined in terms of the torsion tensor constructed from the Cartan connection. The torsion tensor is $T_{\mu \lambda \nu}=e_{a \mu} T_{\lambda \nu}^{a}$, with

$$
T_{\lambda \nu}^{a}=\partial_{\lambda} e^{a}{ }_{\nu}-\partial_{\nu} e^{a}{ }_{\lambda}
$$

The curvature tensor obtained from $\Gamma_{\mu \lambda \nu}$ is identically zero which, using (5), leads to

$$
e R(e) \equiv-e\left(\frac{1}{4} T^{a b c} T_{a b c}+\frac{1}{2} T^{a b c} T_{b a c}-T^{a} T_{a}\right)+2 \partial_{\mu}\left(e T^{\mu}\right),
$$

where $R(e)$ is the scalar curvature of a Riemannian manifold in terms of the tetrad field and $T^{\mu}=T^{b}{ }_{b}{ }^{\mu}$. Since the divergence term in (8) does not contribute to the field equations, the teleparallel lagrangian density is

$$
\begin{aligned}
\mathfrak{L}\left(e_{a \mu}\right) & =-\kappa e\left(\frac{1}{4} T^{a b c} T_{a b c}+\frac{1}{2} T^{a b c} T_{b a c}-T^{a} T_{a}\right)-\mathfrak{Q}_{M} \\
& \equiv-\kappa e \Sigma^{a b c} T_{a b c}-\mathfrak{Q}_{M},
\end{aligned}
$$


where $\kappa=1 /(16 \pi), \mathfrak{Q}_{M}$ is the Lagrangian density of matter fields, and $\Sigma^{a b c}$ is given by

$$
\Sigma^{a b c}=\frac{1}{4}\left(T^{a b c}+T^{b a c}-T^{c a b}\right)+\frac{1}{2}\left(\eta^{a c} T^{b}-\eta^{a b} T^{c}\right),
$$

with $T^{a}=e^{a}{ }_{\mu} T^{\mu}$. It is important to note that the EinsteinHilbert Lagrangian density is equivalent to its teleparallel version given by (9). Thus both theories share the same results concerning dynamics and, up to now, observational data.

Performing a variational derivative of the Lagrangian density with respect to $e^{a \mu}$, which are the dynamical variables of the system, the field equations are

$$
e_{a \lambda} e_{b \mu} \partial_{\nu}\left(e \Sigma^{b \lambda \nu}\right)-e\left(\Sigma^{b v}{ }_{a} T_{b \nu \mu}-\frac{1}{4} e_{a \mu} T_{b c d} \Sigma^{b c d}\right)=\frac{1}{4 \kappa} e T_{a \mu},
$$

where $T_{a \mu}=e_{a}{ }^{\lambda} T_{\mu \lambda}=(1 / e)\left(\delta \mathscr{L}_{M} / \delta e^{a \mu}\right)$ is the energymomentum tensor of matter fields. It is possible to show by explicit calculations the equivalence of (11) and Einstein equations [26].

The field equations can be rewritten as

$$
\partial_{\nu}\left(e \Sigma^{a \lambda \nu}\right)=\frac{1}{4 \kappa} e e_{\mu}^{a}\left(t^{\lambda \mu}+T^{\lambda \mu}\right)
$$

where $t^{\lambda \mu}$ is defined by

$$
t^{\lambda \mu}=\kappa\left(4 \Sigma^{b c \lambda} T_{b c}{ }^{\mu}-g^{\lambda \mu} \Sigma^{b c d} T_{b c d}\right)
$$

Since $\Sigma^{a \lambda v}$ is skew symmetric in the last two indices, it follows that

$$
\partial_{\lambda} \partial_{\nu}\left(e \Sigma^{a \lambda \nu}\right) \equiv 0
$$

Thus we get

$$
\partial_{\lambda}\left(e t^{a \lambda}+e T^{a \lambda}\right)=0
$$

which yields the continuity equation:

$$
\begin{aligned}
& \frac{d}{d t} \int_{V} d^{3} x e e_{\mu}^{a}\left(t^{0 \mu}+T^{0 \mu}\right) \\
& \quad=-\oint_{S} d S_{j}\left[e e^{a}{ }_{\mu}\left(t^{j \mu}+T^{j \mu}\right)\right] .
\end{aligned}
$$

It should be noted that the above expression works as a conservation law for the sum of energy-momentum tensor of matter fields and for the quantity $t^{\lambda \mu}$. Thus $t^{\lambda \mu}$ is interpreted as the energy momentum tensor of the gravitational field [27]. Therefore, one can write the total energy-momentum contained in a three-dimensional volume $V$ of space as

$$
P^{a}=\int_{V} d^{3} x e e^{a}{ }_{\mu}\left(t^{0 \mu}+T^{0 \mu}\right) .
$$

It is worth noting that the above expression is invariant under coordinate transformation and transforms like a vector under Lorentz transformations. Such features are desirable and expected for a true energy-momentum vector.

\section{Noncommutative Corrections for the Gravitational Energy in Schwarzschild Spacetime}

In this section we will start with Schwarzschild spacetime [28], described by the following line element:

$$
\begin{aligned}
d s^{2}= & -\left(1-\frac{2 M}{r}\right) d t^{2}+\left(1-\frac{2 M}{r}\right)^{-1} d r^{2} \\
& +r^{2}\left(d \theta^{2}+\sin ^{2} \theta d \phi^{2}\right),
\end{aligned}
$$

where $M$ is the mass of font. Then a tetrad field adapted to an observer at rest at spatial infinity, which yields the above metric, is

$$
e_{\mu}^{a}=\left[\begin{array}{cccc}
\sqrt{-g_{00}} & 0 & 0 & 0 \\
0 & \sqrt{g_{11}} \sin \theta \cos \phi & \sqrt{g_{22}} \cos \theta \cos \phi & -\sqrt{g_{33}} \sin \phi \\
0 & \sqrt{g_{11}} \sin \theta \sin \phi & \sqrt{g_{22}} \cos \theta \sin \phi & \sqrt{g_{33}} \cos \phi \\
0 & \sqrt{g_{11}} \cos \theta & -\sqrt{g_{22}} \sin \theta & 0
\end{array}\right] .
$$

The noncommutativity is introduced by means of the Moyal product, defined in (3), between two tetrad fields, given by

$$
\widetilde{g}_{\mu \nu}=\frac{1}{2}\left(e^{a}{ }_{\mu} \star e_{a \nu}+e^{a}{ }_{\nu} \star e_{a \mu}\right)
$$

Such a procedure could seem ad hoc; however this is well established; see, for instance, [29]. It constitutes a deformed algebra of diffeomorphisms which modifies only the Leibniz rule, leaving the other quantities unaltered. Although we use the same relation between the new metric tensor and the tetrad field, we point out that our formalism is focused on the teleparallel version in which realm is possible to define and give the corrections of the gravitational energy. Thus the new components of the metric tensor due to the noncommutativity of spacetime are written in terms of the old ones, up to the second order in $\theta^{\mu \nu}$, as

$$
\begin{gathered}
\tilde{g}_{00}=g_{00}, \\
\widetilde{g}_{11}=g_{11}+\frac{1}{4} \theta^{23}\left[\theta^{23} g_{11} \cos (2 \theta)+\theta^{13} \sin (2 \theta)\left(\frac{d g_{11}}{d r}\right)\right] \\
+\frac{1}{8}\left[\left(\theta^{13} \sin \theta\right)^{2}+\left(\theta^{12}\right)^{2}\right]\left(\frac{d^{2} g_{11}}{d r^{2}}\right), \\
\tilde{g}_{22}=g_{22}-\frac{1}{4} \theta^{23}\left[\theta^{23} g_{22} \cos (2 \theta)+\theta^{13} \sin (2 \theta)\left(\frac{d g_{22}}{d r}\right)\right] \\
+\frac{1}{8}\left[\left(\theta^{13} \cos \theta\right)^{2}+\left(\theta^{12}\right)^{2}\right]\left(\frac{d^{2} g_{22}}{d r^{2}}\right),
\end{gathered}
$$




$$
\begin{aligned}
& \tilde{g}_{33}=g_{33}+\frac{1}{8}\left(\theta^{13} \frac{\partial}{\partial r}+\theta^{23} \frac{\partial}{\partial \theta}\right)^{2} g_{33} \\
& +\frac{\left(\theta^{12}\right)^{2}}{32 g_{33}^{2}}\left[\left(\frac{\partial^{2} g_{33}}{\partial \theta^{2}}\right)\left(\frac{\partial g_{33}}{\partial r}\right)^{2}+2 g_{33}\left(\frac{\partial^{2} g_{33}}{\partial \theta \partial r}\right)^{2}\right. \\
& +\left(\frac{\partial g_{33}}{\partial \theta}\right)^{2}\left(\frac{\partial^{2} g_{33}}{\partial r^{2}}\right) \\
& -2 g_{33}\left(\frac{\partial^{2} g_{33}}{\partial \theta^{2}}\right)\left(\frac{\partial^{2} g_{33}}{\partial r^{2}}\right) \\
& \left.-2\left(\frac{\partial g_{33}}{\partial \theta}\right)\left(\frac{\partial g_{33}}{\partial r}\right)\left(\frac{\partial^{2} g_{33}}{\partial \theta \partial r}\right)\right], \\
& \tilde{g}_{12}=\left(\frac{\sin (2 \theta)}{32 g_{22}^{3 / 2} g_{11}^{3 / 2}}\right) \\
& \times\left\{-\frac{1}{2}\left(\theta^{13}\right)^{2}\left[g_{22}\left(\frac{d g_{11}}{d r}\right)-g_{11}\left(\frac{d g_{22}}{d r}\right)\right]^{2}\right. \\
& +\left(\theta^{13}\right)^{2} g_{11} g_{22}\left[g_{22}\left(\frac{d^{2} g_{11}}{d r^{2}}\right)+g_{11}\left(\frac{d^{2} g_{22}}{d r^{2}}\right)\right] \\
& -8\left(\theta^{23}\right)^{2} g_{11}^{2} g_{22}^{2} \\
& \left.+4 \theta^{13} \theta^{23} g_{11} g_{22} \cot (2 \theta)\left[\frac{d}{d r}\left(g_{22} g_{11}\right)\right]\right\}, \\
& \widetilde{g}_{13}=\left(\frac{\theta^{12}}{16 g_{33}^{3 / 2} g_{11}^{1 / 2}}\right) \\
& \times\left\{\theta ^ { 2 3 } \left[\operatorname { c o s } \theta \left(\left(\frac{d g_{11}}{d r}\right)\left(\frac{\partial g_{33}}{\partial \theta}\right) g_{33}\right.\right.\right. \\
& -2 g_{11}\left(\frac{\partial^{2} g_{33}}{\partial \theta \partial r}\right) g_{33} \\
& \left.+g_{11}\left(\frac{\partial g_{33}}{\partial \theta}\right)\left(\frac{\partial g_{33}}{\partial r}\right)\right) \\
& +\sin \theta\left(-\frac{1}{2}\left(\frac{d g_{11}}{d r}\right)\left(\frac{\partial g_{33}}{\partial \theta}\right)^{2}\right. \\
& +g_{33}\left(\frac{d g_{11}}{d r}\right)\left(\frac{\partial^{2} g_{33}}{\partial \theta^{2}}\right) \\
& \left.\left.+2 g_{11} g_{33}\left(\frac{\partial g_{33}}{\partial r}\right)\right)\right] \\
& +\theta^{13}\left[\operatorname { c o s } \theta \left(-g_{33}\left(\frac{d g_{11}}{d r}\right)\left(\frac{\partial g_{33}}{\partial r}\right)\right.\right. \\
& +g_{11}\left(\frac{\partial g_{33}}{\partial r}\right)^{2}
\end{aligned}
$$

$$
\begin{aligned}
& \left.-2 g_{11} g_{33}\left(\frac{\partial^{2} g_{33}}{\partial r^{2}}\right)\right)+\sin \theta \\
& \cdot\left(-\frac{1}{2}\left(\frac{d g_{11}}{d r}\right)\left(\frac{\partial g_{33}}{\partial \theta}\right)\left(\frac{\partial g_{33}}{\partial r}\right)\right. \\
& +g_{33} \frac{\partial}{\partial r}\left(\frac{\partial g_{33}}{\partial \theta} \cdot \frac{d g_{11}}{d r}\right) \\
& \left.\left.\left.-\frac{1}{2}\left(\frac{g_{33}}{g_{11}}\right)\left(\frac{\partial g_{33}}{\partial \theta}\right)\left(\frac{d g_{11}}{d r}\right)^{2}\right)\right]\right\}, \\
& \tilde{g}_{23}=\left(\frac{\theta^{12}}{32 g_{33}^{3 / 2} g_{22}^{3 / 2}}\right) \\
& \times\left[4 \theta^{13} g_{22}^{2} g_{33} \sin \theta\left(\frac{\partial^{2} g_{33}}{\partial r^{2}}\right)\right. \\
& -\theta^{13} g_{33} \cos \theta\left(\frac{\partial g_{33}}{\partial \theta}\right)\left(\frac{d g_{22}}{d r}\right)^{2} \\
& +2 \theta^{13} g_{33} g_{22} \cos \theta\left(\frac{\partial g_{33}}{\partial \theta}\right)\left(\frac{d^{2} g_{22}}{d r^{2}}\right) \\
& +4 \theta^{23} g_{22}^{2} g_{33} \cos \theta\left(\frac{\partial g_{33}}{\partial r}\right) \\
& -2 \theta^{13} g_{22}^{2} \sin \theta\left(\frac{\partial g_{33}}{\partial r}\right)^{2} \\
& -2 \theta^{23} g_{22}^{2} \sin \theta\left(\frac{\partial g_{33}}{\partial \theta}\right)\left(\frac{\partial g_{33}}{\partial r}\right) \\
& -2 \theta^{23} g_{33} g_{22} \sin \theta\left(\frac{d g_{22}}{d r}\right)\left(\frac{\partial g_{33}}{\partial \theta}\right) \\
& -\theta^{23} g_{22} \cos \theta\left(\frac{d g_{22}}{d r}\right) \cdot\left(\frac{\partial g_{33}}{\partial \theta}\right)^{2} \\
& +2 \theta^{23} g_{33} g_{22} \cos \theta\left(\frac{d g_{22}}{d r}\right)\left(\frac{\partial^{2} g_{33}}{\partial \theta^{2}}\right) \\
& +2 \theta^{13} g_{33} g_{22} \sin \theta\left(\frac{d g_{22}}{d r}\right)\left(\frac{\partial g_{33}}{\partial r}\right) \\
& -\theta^{13} g_{22} \cos \theta\left(\frac{d g_{22}}{d r}\right)\left(\frac{\partial g_{33}}{\partial \theta}\right)\left(\frac{\partial g_{33}}{\partial r}\right) \\
& +2 \theta^{13} g_{22} g_{33} \cos \theta\left(\frac{d g_{22}}{d r}\right)\left(\frac{\partial^{2} g_{33}}{\partial \theta \partial r}\right) \\
& \left.+4 \theta^{23} g_{22}^{2} g_{33} \sin \theta\left(\frac{\partial^{2} g_{33}}{\partial \theta \partial r}\right)\right] \text {. }
\end{aligned}
$$

It should be noted that such components are always quadratic in the noncommutative parameter $\theta^{i j}$ which was first decisively pointed out in [30] and corroborated in [31]. This indicates that the corrections have very tiny values. Therefore the new metric tensor obeys approximately Einstein's equations. We point out that the corrections above are different from the ones presented in [31], for a great review we recommend [32]. 
Now we have to write another tetrad field for the new components of the metric tensor, but still adapted to a rest frame, which is the referential frame we would like to analyze the problem of the gravitational energy. This means that we suppose that a relation exists between the spacetime which is defined by the corrected metric tensor and a Weitzenböck spacetime. Indeed we are assuming that the Lorentz symmetry still holds. We, in this sense, assume the following correspondence $\widetilde{g}_{\mu \nu}=\widetilde{e}_{\mu}^{a} \widetilde{e}_{a v}$, where $\widetilde{e}_{\mu}^{a}$ is given by

$$
\widetilde{e}_{\mu}^{a}=\left[\begin{array}{cccc}
A & 0 & 0 & 0 \\
0 & B \sin \theta \cos \phi+E r \cos \theta \cos \phi-F r \sin \theta \sin \phi & C r \cos \theta \cos \phi-G r \sin \theta \sin \phi & -H r \sin \theta \sin \phi \\
0 & B \sin \theta \sin \phi+E r \cos \theta \sin \phi+F r \sin \theta \cos \phi & C r \cos \theta \sin \phi+G r \sin \theta \cos \phi & H r \sin \theta \cos \phi \\
0 & B \cos \theta-E r \sin \theta & -C r \sin \theta & 0
\end{array}\right],
$$

where

$$
\begin{gathered}
A=\sqrt{-\widetilde{g}_{00}}, \\
B=\frac{\delta_{1}}{\delta}, \\
C=\frac{\delta}{r \sqrt{\widetilde{g}_{33}}}, \\
E=\frac{\widetilde{g}_{12} \widetilde{g}_{33}-\widetilde{g}_{23} \widetilde{g}_{13}}{r \sqrt{\widetilde{g}_{33}} \delta}, \\
F=\frac{\widetilde{g}_{13}}{\sqrt{\widetilde{g}_{33}} r \sin \theta}, \\
G=\frac{\widetilde{g}_{23}}{\sqrt{\widetilde{g}_{33}} r \sin \theta}, \\
H=\frac{\sqrt{\widetilde{g}_{33}}}{r \sin \theta} .
\end{gathered}
$$

The quantities $\delta$ and $\delta_{1}$ are defined by

$$
\begin{gathered}
\delta^{2}=\widetilde{g}_{22} \widetilde{g}_{33}-\widetilde{g}_{23}^{2}, \\
\delta_{1}^{2}=\widetilde{g}_{11} \delta^{2}-\widetilde{g}_{22} \widetilde{g}_{13}^{2}-\widetilde{g}_{33} \widetilde{g}_{12}^{2}+2 \widetilde{g}_{12} \widetilde{g}_{23} \widetilde{g}_{13} .
\end{gathered}
$$

At this point we would like to clarify some aspects. Firstly we are not dealing with a full noncommutative theory, but we are rather looking for some corrections of the classical theory. In this sense the noncommutativity was introduced in order to construct the corrected metric tensor, and then we assume the existence of a Weitzenböck spacetime which matches such a metric tensor. In this context the tetrad field $\tilde{e}^{a}{ }_{\mu}$ is classical since it is induced by the new metric tensor. Thus we can use the prescriptions of teleparallel gravity to work on the corrections of the gravitational energy. In order to compute such a quantity, the relevant nonvanishing components of torsion tensor are

$$
\begin{gathered}
\widetilde{T}_{112}=-\frac{1}{2}\left(\frac{\partial \widetilde{g}_{11}}{\partial \theta}\right) \\
+\frac{1}{2 \delta^{2}}\left[\alpha\left(\frac{\partial \widetilde{g}_{22}}{\partial r}\right)+\beta\left(\frac{\widetilde{g}_{23}}{\widetilde{g}_{33}}\right)\left(\frac{\partial \widetilde{g}_{33}}{\partial r}\right)\right. \\
\left.-2 \beta\left(\frac{\partial \widetilde{g}_{23}}{\partial r}\right)\right],
\end{gathered}
$$

$$
\begin{aligned}
& \widetilde{T}_{212}=\frac{1}{2}\left(\frac{\partial \widetilde{g}_{22}}{\partial r}\right)-\left(\frac{\partial \widetilde{g}_{12}}{\partial \theta}\right)-\delta_{1} \widetilde{g}_{33}^{-1 / 2} \\
& +\frac{1}{2 \delta^{2}}\left[\alpha\left(\frac{\partial \widetilde{g}_{22}}{\partial \theta}\right)+\beta\left(\frac{\widetilde{g}_{23}}{\widetilde{g}_{33}}\right)\left(\frac{\partial \widetilde{g}_{33}}{\partial \theta}\right)\right. \\
& \left.-2 \beta\left(\frac{\partial \widetilde{g}_{22}}{\partial \theta}\right)\right], \\
& \widetilde{T}_{312}=\frac{1}{2 \widetilde{g}_{33}}\left[2 \widetilde{g}_{33}\left(\frac{\partial \widetilde{g}_{23}}{\partial r}\right)-\widetilde{g}_{23}\left(\frac{\partial \widetilde{g}_{33}}{\partial r}\right)\right. \\
& \left.+\widetilde{g}_{13}\left(\frac{\partial \widetilde{g}_{33}}{\partial \theta}\right)-2 \widetilde{g}_{33}\left(\frac{\partial \widetilde{g}_{13}}{\partial \theta}\right)\right], \\
& \widetilde{T}_{113}=\frac{1}{2}\left(\frac{\widetilde{g}_{13}}{\widetilde{g}_{33}}\right)\left(\frac{\partial \widetilde{g}_{33}}{\partial r}\right), \\
& \widetilde{T}_{213}=-\frac{1}{2 \widetilde{g}_{33} \delta}\left[2 \widetilde{g}_{33} \beta \cos \theta+2 \widetilde{g}_{23} \widetilde{g}_{33}^{1 / 2}-\widetilde{g}_{23} \delta\left(\frac{\partial \widetilde{g}_{33}}{\partial r}\right)\right], \\
& \widetilde{T}_{313}=\frac{1}{2}\left(\frac{\partial \widetilde{g}_{33}}{\partial r}\right)-\frac{1}{\delta}\left(\alpha \cos \theta+\delta_{1} \tilde{g}_{33}^{1 / 2} \sin \theta\right), \\
& \widetilde{T}_{123}=-\frac{1}{2}\left(\frac{\widetilde{g}_{13}}{\widetilde{g}_{33}}\right)\left(\frac{\partial \widetilde{g}_{33}}{\partial \theta}\right)-\frac{1}{\delta}\left(\beta \cos \theta+\delta_{1} \widetilde{g}_{23} \widetilde{g}_{33}^{-1 / 2} \sin \theta\right) \text {, } \\
& \widetilde{T}_{223}=\frac{1}{2}\left(\frac{\widetilde{g}_{23}}{\widetilde{g}_{33}}\right)\left(\frac{\partial \widetilde{g}_{33}}{\partial \theta}\right), \\
& \widetilde{T}_{323}=\frac{1}{2}\left(\frac{\partial \widetilde{g}_{33}}{\partial \theta}\right)-\delta \cos \theta,
\end{aligned}
$$

where

$$
\begin{aligned}
& \alpha=\widetilde{g}_{33} \widetilde{g}_{12}-\widetilde{g}_{23} \widetilde{g}_{13}, \\
& \beta=\widetilde{g}_{12} \widetilde{g}_{23}-\widetilde{g}_{22} \widetilde{g}_{13} .
\end{aligned}
$$

Using (10), it yields

$$
\begin{aligned}
\widetilde{\Sigma}^{001}= & \frac{1}{2\left(-\widetilde{g}_{00}\right) \delta_{1}^{2}} \\
& \times\left[-\widetilde{T}_{112}\left(\delta^{2} \widetilde{g}_{12}+\alpha \widetilde{g}_{11}\right)\right.
\end{aligned}
$$




$$
\begin{aligned}
& -\widetilde{T}_{212}\left(\delta^{2} \widetilde{g}_{22}+\alpha \widetilde{g}_{12}\right)-\widetilde{T}_{312}\left(\delta^{2} \widetilde{g}_{23}+\alpha \widetilde{g}_{12}\right) \\
& -\widetilde{T}_{113}\left(\delta^{2} \widetilde{g}_{13}-\beta \widetilde{g}_{11}\right)-\widetilde{T}_{213}\left(\delta^{2} \widetilde{g}_{23}-\beta \widetilde{g}_{12}\right) \\
& -\widetilde{T}_{313}\left(\delta^{2} \widetilde{g}_{33}-\beta \widetilde{g}_{13}\right)+\widetilde{T}_{123}\left(\alpha \widetilde{g}_{13}+\beta \widetilde{g}_{12}\right) \\
& \left.+\widetilde{T}_{223}\left(\alpha \widetilde{g}_{23}+\beta \widetilde{g}_{22}\right)+\widetilde{T}_{323}\left(\alpha \widetilde{g}_{33}+\beta \widetilde{g}_{23}\right)\right],
\end{aligned}
$$

then it is possible to find $\widetilde{\Sigma}^{(0) 01}=\widetilde{e}^{(0)}{ }_{0} \widetilde{\Sigma}^{001}$, which after some algebraic manipulations, is written as

$$
\begin{aligned}
4 e \widetilde{\Sigma}^{(0) 01}=\left(\frac{2}{\delta_{1}}\right)\{ & \widetilde{g}_{33}^{1 / 2} \delta_{1}+\left(\frac{\delta \delta_{1}}{\sqrt{\widetilde{g}_{33}}}\right) \sin \theta-\frac{1}{2} \frac{\partial}{\partial r}\left(\widetilde{g}_{33} \widetilde{g}_{22}\right) \\
& +\frac{\partial}{\partial \theta}\left(\widetilde{g}_{33} \widetilde{g}_{12}\right) \\
& \left.+\widetilde{g}_{23}\left[\left(\frac{\partial \widetilde{g}_{23}}{\partial r}\right)-\left(\frac{\partial \widetilde{g}_{13}}{\partial \theta}\right)\right]\right\}
\end{aligned}
$$

$$
\begin{aligned}
+\left(\frac{1}{\delta^{2} \delta_{1}}\right) & \left\{\left(\widetilde{g}_{23} \widetilde{g}_{13}-\widetilde{g}_{12} \widetilde{g}_{33}\right)\left[\frac{\partial\left(\widetilde{g}_{22} \widetilde{g}_{33}\right)}{\partial \theta}\right]\right. \\
& \left.+2 \widetilde{g}_{33}\left(\widetilde{g}_{12} \widetilde{g}_{23}-\widetilde{g}_{22} \widetilde{g}_{13}\right)\left(\frac{\partial \widetilde{g}_{23}}{\partial \theta}\right)\right\} .
\end{aligned}
$$

Then taking the limit $r \rightarrow \infty$ it yields

$$
\begin{aligned}
4 e \widetilde{\Sigma}^{(0) 01}= & 4 M \sin \theta-\left(\theta^{23}\right)^{2}\left(\frac{\cos ^{2} \theta}{\sin \theta}\right) \\
& \times\left[M\left(\frac{11}{8}+\cos ^{2} \theta\right)+\lim _{r \rightarrow \infty} \frac{3}{2} r\left(\frac{1}{4}+\cos ^{2} \theta\right)\right] .
\end{aligned}
$$

The energy is calculated using (17) and performing a regularization procedure in order to avoid divergences. Such a regularization procedure is necessary mainly because the limit of $\tilde{g}_{\mu \nu}$ for $r \rightarrow \infty$ does not lead to Minkowski metric tensor: instead it produces (in spherical coordinates)

$$
\widetilde{\eta}_{\mu \nu}=\left(\begin{array}{cccc}
-1 & 0 & 0 & 0 \\
0 & 1+\frac{\left(\theta^{23}\right)^{2} \cos (2 \theta)}{4} & -\frac{\left(\theta^{23}\right)^{2} r \sin (2 \theta)}{4} & \frac{\theta^{12} \theta^{23}\left(\sin ^{2} \theta-\cos \theta\right)}{4} \\
0 & -\frac{\left(\theta^{23}\right)^{2} r \sin (2 \theta)}{4} & {\left[1-\frac{\left(\theta^{23}\right)^{2} \cos (2 \theta)}{4}\right] r^{2}} & 0 \\
0 & \frac{\theta^{12} \theta^{23}\left(\sin ^{2} \theta-\cos \theta\right)}{4} & 0
\end{array}\right],
$$

which generates nonvanishing components of the torsion tensor. This procedure consists of subtracting from the quantity analyzed what stands when a physical parameter, such as the mass of the black hole, is set to zero. Hence we get

$$
\widetilde{P}^{(0)}=M+\frac{1}{4}\left(\frac{49}{3}-\frac{15}{2} \ln 2\right)\left(\theta^{23}\right)^{2} M,
$$

thus the correction in the gravitational energy due to the noncommutativity of spacetime is

$$
\Delta P^{(0)}=\frac{1}{4}\left(\frac{49}{3}-\frac{15}{2} \ln 2\right)\left(\theta^{23}\right)^{2} M .
$$

Alternatively the need for a regularization procedure could be contoured by means of the choice $\theta^{23}=0$, in such a way there would be no correction for the Schwarzschild energy.

\section{Conclusion}

In this work we start with Schwarzschild spacetime, and then we give the corrections due to the noncommutativity of spacetime. Here it is introduced by replacing the normal product between tetrads by the Moyal product, rather than applying such a procedure in lagrangian density. This approach is well known in the literature to predict some noncommutative corrections in the metric tensor. The new metric tensor leads to a new tetrad field which is used to calculate the gravitational energy of spacetime. It is well known that the energy of Schwarzschild spacetime is equal to $M$; therefore we get a correction in the energy equal to $\Delta P^{(0)}$. We stress out that the expression for the gravitational field has been developed and tested over the years in the context of TEGR. Since the noncommutative parameter is arbitrary (it should be given by experimental data), we speculate that such a correction in the gravitational energy can be associated to quantum effects in the realm of gravitational field. If the correction represents the energy of gravitons, then it should be proportional to the Planck's constant. On the other hand the correction is proportional to the mass of the font, which could mean a new kind of quantization associated to the mass of a black hole or a star, for example. This has been expressed in [33]. Therefore the gravitational energy turns out to be of fundamental importance (experimental purposes), since it can tell if the spacetime is commutative or not. For future works we intend to investigate the corrections of the gravitational energy in the context of Kerr spacetime on the 
outer event horizon. We also want to study the solutions of the noncommutative equations that come from the lagrangian density replaced by the Moyal product.

\section{References}

[1] W. Pauli, Scientific Correspondence, vol. 2, Spring, Berlin, Germany, 1985, K. von Meyenn, Ed.

[2] W. Pauli, Scientific Correspondence, vol. 3, Spring, Berlin, Germany, 1993, K. von Meyenn, Ed.

[3] R. Jackiw, "Physical instances of noncommuting coordinates," Nuclear Physics B, vol. 108, pp. 30-36, 2002.

[4] H. S. Snyder, "Quantized space-time," Physical Review, vol. 71, no. 1 , pp. 38-41, 1947.

[5] H. S. Snyder, "The electromagnetic field in quantized spacetime," Physical Review, vol. 72, no. 1, pp. 68-71, 1947.

[6] A. H. Chamseddine, G. Felder, and J. Fröhlich, "Gravity in non-commutative geometry," Communications in Mathematical Physics, vol. 155, no. 1, pp. 205-217, 1993.

[7] W. Kalau and M. Walze, "Gravity, non-commutative geometry and the Wodzicki residue," Journal of Geometry and Physics, vol. 16, no. 4, pp. 327-344, 1995.

[8] D. Kastler, "The dirac operator and gravitation," Communications in Mathematical Physics, vol. 166, no. 3, pp. 633-643, 1995.

[9] A. H. Chamseddine and A. Connes, "The spectral action principle," Communications in Mathematical Physics, vol. 186, no. 3, pp. 731-750, 1997.

[10] A. Connes and J. Lott, "Particle models and noncommutative geometry," Nuclear Physics B, vol. 18, no. 2, pp. 29-47, 1991.

[11] J. C. Vrilly and J. Gracia-Bonda, "Connes' noncommutative differential geometry and the standard model," Journal of Geometry and Physics, vol. 12, pp. 223-301, 1993.

[12] C. Martn, J. Gracia-Bonda, and J. C. Vrilly, “The Standard Model as a noncommutative geometry: the low-energy regime," Physics Reports, vol. 294, pp. 363-406, 1998.

[13] J. Bellissard, A. van Elst, and H. Schulz-Baldes, "The noncommutative geometry of the quantum Hall effect," Journal of Mathematical Physics, vol. 35, no. 10, pp. 5373-5451, 1994.

[14] N. Seiberg and E. Witten, "String theory and noncommutative geometry," Journal of High Energy Physics, vol. 1999, no. 9, article 32, 1999.

[15] E. Akofor, A. P. Balachandran, and A. Joseph, "Quantum fields on the Groenewold-Moyal plane," International Journal of Modern Physics A, vol. 23, no. 11, pp. 1637-1677, 2008.

[16] A. Einstein, "Auf die riemann-metrik und den fernparallelismus gegründete einheitliche feldtheorie," Annals of Mathematics, vol. 102, pp. 685-697, 1930.

[17] E. Cartan, "On a generalization of the notion of reimann curvature and spaces with torsion," in Cosmology and Gravitation: Spin, Torsion, Rotation, and Supergravity, P. G. Bergmann and V. de Sabbata, Eds., vol. 58 of NATO ASI B Proceedings, pp. 489491, 1980.

[18] J. W. Maluf, J. F. da Rocha-Neto, T. M. L. Toribio, and K. H. Castello-Branco, "Energy and angular momentum of the gravitational field in the teleparallel geometry," Physical Review D, vol. 65, no. 12, Article ID 124001, 2002.

[19] A. Komar, "Covariant conservation laws in general relativity," Physical Review, vol. 113, no. 3, pp. 934-936, 1959.

[20] R. Arnowitt, S. Deser, and C. W. Misner, "The dynamics of general relativity," in Gravitation: An Introduction to Current
Research, pp. 227-265, John Wiley \& Sons, New York, NY, USA, 1962.

[21] J. W. Maluf and J. F. da Rocha-Neto, "Hamiltonian formulation of general relativity in the teleparallel geometry," Physical Review D, vol. 64, no. 8, Article ID 084014, 2001.

[22] J. W. Maluf and S. C. Ulhoa, "The energy-momentum of planefronted gravitational waves in the teleparallel equivalent of GR," Physical Review D, vol. 78, Article ID 047502, 2008, Erratum in Physical Review D, vol. 78, Article ID 069901, 2008.

[23] J. Maluf, M. Veiga, and J. da Rocha-Neto, "Regularized expression for the gravitational energy-momentum in teleparallel gravity and the principle of equivalence," General Relativity and Gravitation, vol. 39, pp. 227-240, 2007.

[24] J. F. da Rocha Neto, J. W. Maluf, and S. C. Ulhoa, "Hamiltonian formulation of unimodular gravity in the teleparallel geometry," Physical Review D, vol. 82, no. 12, Article ID 124035, 2010.

[25] S. C. Ulhoa, J. F. da Rocha Neto, and J. W. Maluf, “The gravitational energy problem for cosmological models in teleparallel gravity," International Journal of Modern Physics D, vol. 19, no. 12, pp. 1925-1935, 2010.

[26] J. W. Maluf, "Hamiltonian formulation of the teleparallel description of general relativity," Journal of Mathematical Physics, vol. 35, no. 1, pp. 335-343, 1994.

[27] J. W. Maluf, “The gravitational energy-momentum tensor and the gravitational pressure," Annalen der Physik, vol. 14, no. 11-12, pp. 723-732, 2005.

[28] R. d'Inverno, Introducing Einstein's Relativity, Clarendon Press, Oxford, UK, 4th edition, 1996.

[29] P. Aschieri, C. Blohmann, M. Dimitrijević, F. Meyer, P. Schupp, and J. Wess, "A gravity theory on noncommutative spaces," Classical and Quantum Gravity, vol. 22, no. 17, pp. 3511-3532, 2005.

[30] P. Mukherjee and A. Saha, "Note on the noncommutative correction to gravity," Physical Review D, vol. 74, Article ID 027702, 3 pages, 2006.

[31] M. Chaichian, A. Tureanu, and G. Zet, "Corrections to Schwarzschild solution in noncommutative gauge theory of gravity," Physics Letters B, vol. 660, no. 5, pp. 573-578, 2008.

[32] P. Nicolini, "Noncommutative black holes, the final appeal to quantum gravity: a review," International Journal of Modern Physics A, vol. 24, no. 7, pp. 1229-1308, 2009.

[33] S. C. Ulhoa, "On special requantization of a black hole," Brazilian Journal of Physics, vol. 41, no. 4-6, pp. 309-313, 2011. 

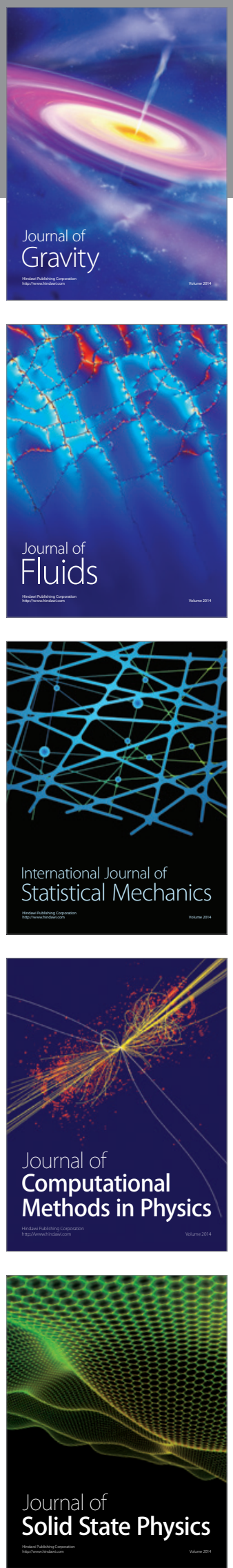

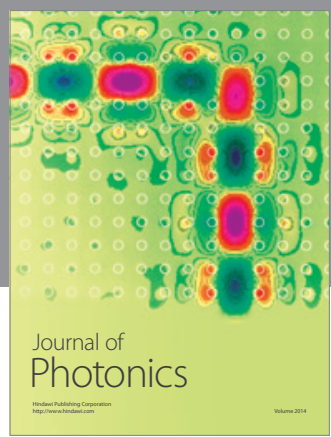

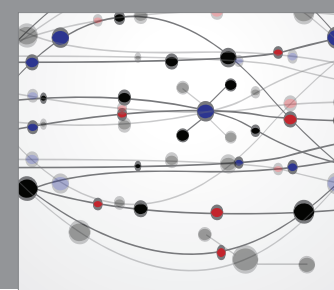

The Scientific World Journal

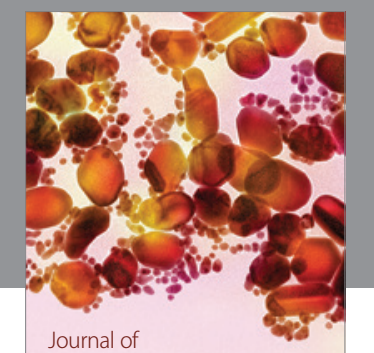

Soft Matter
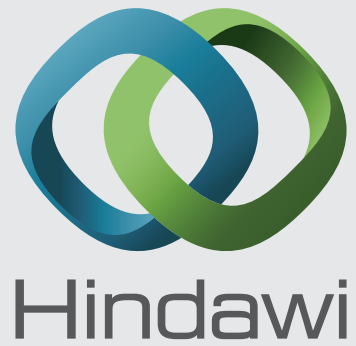

Submit your manuscripts at

http://www.hindawi.com
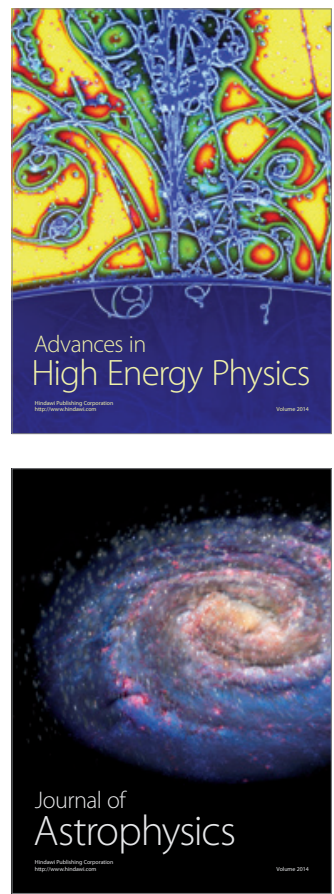
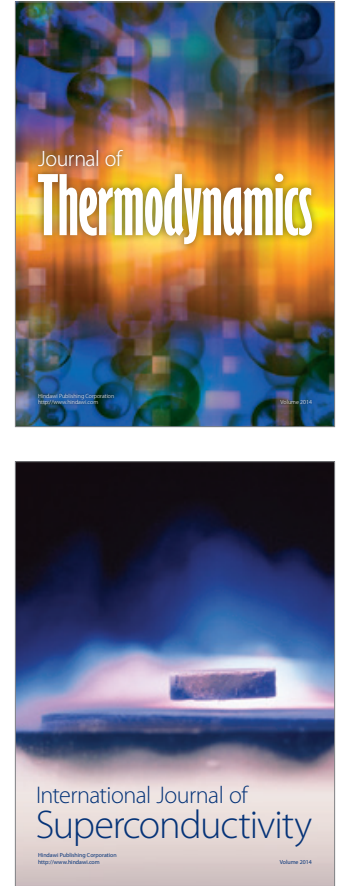
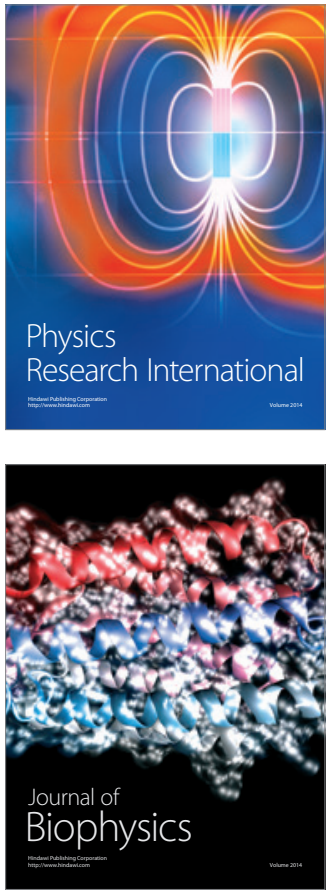
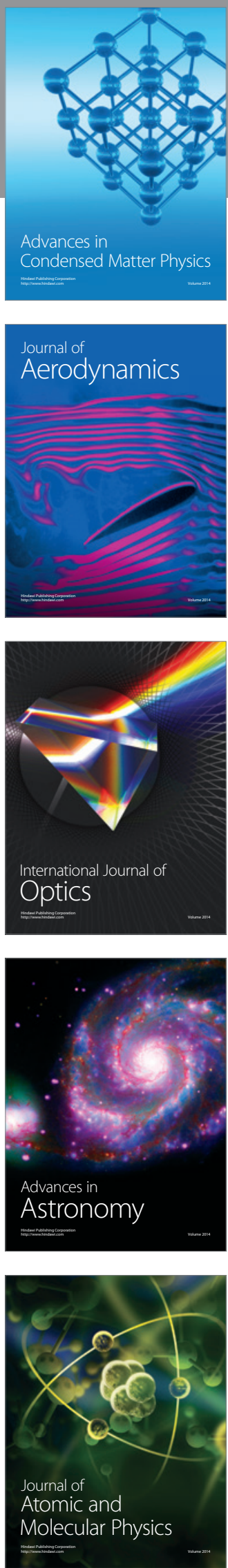